\title{
Existence of multiple equilibrium points in global attractor for damped wave equation
}

Fengjuan Meng ${ }^{1 *}$, Cuncai Liu ${ }^{1}$ and Chang Zhang ${ }^{1}$

"Correspondence:

fjmeng@jsut.edu.cn

'School of Mathematics and

Physics, Jiangsu University of

Technology, Changzhou, China

\begin{abstract}
This paper is a continuation of Meng and Zhong in (Discrete Contin. Dyn. Syst., Ser. B 19:217-230, 2014). We go on studying the property of the global attractor for some damped wave equation with critical exponent. The difference between this paper and Meng and Zhong in (Discrete Contin. Dyn. Syst., Ser. B 19:217-230, 2014) is that the origin is not a local minimum point but rather a saddle point of the Lyapunov function $F$ for the symmetric dynamical systems. Using the abstract result established in Zhang et al. in (Nonlinear Anal., Real World Appl. 36:44-55, 2017), we prove the existence of multiple equilibrium points in the global attractor for some wave equations under some suitable assumptions in the case that the origin is an unstable equilibrium point.
\end{abstract}

MSC: 35L05; 37L05; 35B40; 35B41; 58J20

Keywords: Lyapunov functional; Global attractor; $Z_{2}$ index; Equilibrium points; Wave equation

\section{Introduction}

In this paper, we consider the following weakly damped wave equation:

$$
\begin{cases}u_{t t}+u_{t}-\Delta u-\lambda u+\varphi(u)=0, & (x, t) \in \Omega \times \mathbb{R}^{+}, \\ u=0, & (x, t) \in \partial \Omega \times \mathbb{R}^{+}, \\ u(x, 0)=u_{0}(x), u_{t}(x, 0)=u_{1}(x) & x \in \Omega,\end{cases}
$$

where $\Omega \subset R^{3}$ is a bounded domain with smooth boundary $\partial \Omega$. Let

$$
0<\lambda_{1}<\lambda_{2} \leq \cdots \leq \lambda_{m}<\lambda_{m+1}<\cdots
$$

be the sequence of eigenvalues of $-\Delta$ on $H_{0}^{1}(\Omega)$, and let $e_{j}$ be the eigenfunctions corresponding to $\lambda_{j}, j=1,2, \ldots$. Here we assume that $\lambda \in\left(\lambda_{m}, \lambda_{m+1}\right)$. We also impose the following assumptions on the nonlinear term $\varphi$ :

(A) $\varphi \in C(R)$ is of the form

$$
\varphi(s)=s^{3}-\beta|s|^{\gamma} s,
$$

(c) The Author(s) 2019. This article is distributed under the terms of the Creative Commons Attribution 4.0 International License (http://creativecommons.org/licenses/by/4.0/), which permits unrestricted use, distribution, and reproduction in any medium, provided you give appropriate credit to the original author(s) and the source, provide a link to the Creative Commons license, and indicate if changes were made. 
where $\beta$ is a parameter, and $0<\gamma<2$. The number 3 is called the critical exponent, since the nonlinearity $f$ is not compact in this case.

The existence of the global attractor (see Definition 2.2) for wave equations like (1.1) has been studied extensively by many authors; we refer to $[1,2,11,14]$ and the references therein for more detail.

We now want to examine the attractor itself in more detail. In concise, we study the properties of the global attractor and obtain the existence of multiple equilibrium points (see Def. 2.6) in the global attractor in $H_{0}^{1}(\Omega) \times L^{2}(\Omega)$.

It is well known that if a system or a semigroup $\{S(t)\}_{t \geq 0}$ has a global attractor $\mathcal{A}$ in a Banach space $X$ and has a Lyapunov function $F$ (see Def. 2.5) on some neighborhood of $\mathcal{A}$, then

$$
\mathcal{A}=W^{u}(\mathcal{E})
$$

where $\mathcal{E}$ is the set of all fixed points, and $W^{u}(\mathcal{E})$ is the unstable manifold (see Def. 2.8) of $\mathcal{E}$. Furthermore, if $\mathcal{E}$ is discrete, then

$$
\mathcal{A}=W^{u}(\mathcal{E})=\bigcup_{z \in \mathcal{E}} W^{u}(z)
$$

(see $[12,14]$ etc.). On the other hand, all complete bounded orbits lie in the global attractor $\mathcal{A}$ (see[12] etc.), that is,

$$
\left\{\theta(t): \theta(t) \text { is a complete bounded orbit of }\{S(t)\}_{t \geq 0}\right\} \subset \mathcal{A} \text {. }
$$

We also note that the global attractor is connected if the phase space is connected (see $[12,14]$ etc). As the phase spaces we consider are usually Hilbert or Banach spaces, the global attractor is connected.

In this case, each complete bounded orbit $\theta(t)$ is always connected to some pair of fixed points of a semigroup $\{S(t)\}_{t \geq 0}$, and $\theta(t)$ is contained in the unstable manifold from the one fixed point (see Def. 2.6) and the stable manifold (see Def. 2.7) from the other fixed point. From this point of view, if the number of fixed points in $\mathcal{A}$ is large, then the structure of such an attractor can be completely specified by a list of fixed points that are joined to each other. Thus, it is meaningful for us to investigate the multiplicity of equilibrium points in the global attractor $\mathcal{A}$.

To the best of our knowledge, there are few related results. Fortunately, recently, the authors in [18] and [16] established the criterions to show the existence of the multiplicity of equilibrium points under some proper conditions.

In detail, if the semigroup $\{S(t)\}_{t \geq 0}$ is odd and the Lyapunov function $F$ is even, and if the origin is a strictly local minimum point of $F$, then the authors in [18] have proved the existence of the multiple equilibrium points in the global attractors for the symmetric dynamical systems by estimating the lower bound of $Z_{2}$ index of two disjoint subsets of the global attractor for which one subset is located in the area where the Lyapunov function $F$ is positive and the other subset is located in the area where the Lyapunov function $F$ is negative. As applications, the authors have considered the reaction-diffusion equations [18], wave equations [8, 9], and $p$-Laplacian equation [15] and proved that the corresponding 
semigroups $\{S(t)\}_{t \geq 0}$ possess at least $2 n$ pairs of different fixed points in the global attractor $\mathcal{A}$.

On the other hand, if the origin is no longer a strictly local minimum point but a saddle point of $F$, then we cannot estimate the lower bound of $Z_{2}$ index of the global attractor by the method or technique in [18]. Fortunately, in [16], by proving a new lemma, which is analogous to the intersection lemma in [13], we can still prove the existence of multiple fixed points in a global attractor for some symmetric semigroups with a Lyapunov function $F$. To be precise, in [16] the authors have mainly obtained the following results.

Theorem 1.1 Let $X$ be a Banach space, and let $\{S(t)\}_{t \geq 0}$ be a continuous semigroup on $X$. Assume that $\{S(t)\}_{t \geq 0}$ satisfies the following conditions:

$\left(A_{1}^{\prime}\right) S(t): X \rightarrow X$ is odd for each $t \geq 0$,

$\left(A_{2}^{\prime}\right)\{S(t)\}_{t \geq 0}$ possesses a global attractor $\mathcal{A}$ in $X$,

$\left(A_{3}^{\prime}\right)\{S(t)\}_{t \geq 0}$ has a $C^{0}$ even Lyapunov function $F$ on $X$,

$\left(A_{4}^{\prime}\right)$ There exist two closed subspaces $X^{+}$and $X^{-}$of $X$ satisfying

$\left(A_{4}^{\prime}-i\right) \operatorname{codim} X^{+} \leq \operatorname{dim} X^{-}<\infty$ and $X=X^{+}+X^{-}$, and

$\left(A_{4}^{\prime}-i i\right)$ there exist two positive constants $\alpha$ and $\varrho$ such that

$$
\left.F\right|_{X^{+} \cap \partial B(0, \delta)} \geq \alpha,
$$

where $B(0, \varrho)$ is a ball of radius $\varrho$ in $X$ with center at the origin,

$\left(A_{4}^{\prime}-\right.$ iii) there exist two positive constants $R$ and $0<\rho<R$ such that

$$
\left.F\right|_{X^{-} \cap \partial B(0, R)}<\inf _{v \in \partial B(0, \rho)} F(v)<F(0)=0 .
$$

Then we have:

(i)

$$
\gamma\left(\mathcal{A} \cap F^{-1}((-\infty, 0])\right) \geq \operatorname{dim} X^{-}
$$

where $\delta=\inf _{v \in \partial B(0, \rho)} F(v)<0$ and $F^{-1}((-\infty, \delta])=\{u \in X: F(u) \leq \delta\}$,

(ii)

$$
\begin{aligned}
& \qquad \gamma\left(\mathcal{A} \cap F^{-1}([\alpha, \infty))\right) \geq \operatorname{dim} X^{-}-\operatorname{codim} X^{+}, \\
& \text {where } F^{-1}([\alpha, \infty))=\{u \in X: \alpha \leq F(u)<\infty\} .
\end{aligned}
$$

Theorem 1.2 Let $\{S(t)\}_{t \geq 0}$ be a continuous semigroup on $X$. Under the assumptions of Theorem 1.1, the semigroup $\{S(t)\}_{t \geq 0}$ possesses at least $\operatorname{dim} X^{-}-\operatorname{codim} X^{+}$pairs of different fixed points in $\mathcal{A} \cap F^{-1}((0, \infty))$.

As an application of Theorems 1.1 and 1.2, the authors have considered the reactiondiffusion equations in [16] and the $p$-Laplacian equation in [7].

The current paper is mostly related to [9] and motivated by [16]. In [9] the authors have proved the existences of multiple equilibrium points in the global attractor of (1.1) with $\lambda=0$. The essential difference between this paper and [9] is that the origin is not a local 
minimum point but a saddle point of the corresponding Lyapunov function. Also, compared with the reaction-diffusion equations in [16], the phase space of the wave equation is the product space $H_{0}^{1}(\Omega) \times L^{2}(\Omega)$; nevertheless, to apply Theorem 1.1, one key point is to decompose the phrase space, and we present a new strategy to decompose the product space (see the proof of Theorem 3.3 in detail).

The rest of the paper is organized as follows. In the next section, for the convenience of the reader, we provide some preliminaries. In Sect. 3, by applying Theorems 1.1 and 1.2 , we consider the existence of multiple stationary solutions for some symmetric wave equation with weak damping.

Throughout this paper, $X$ is a Banach space endowed with norm $\|\cdot\|_{X}$, and $C$ is any positive constant which may be different from line to line and even in the same line.

\section{Preliminaries}

We first give some basic definitions about semigroups and global attractors, which can be found in $[1,4,10,12,14]$ and references therein.

Definition 2.1 Let $\{S(t)\}_{t \geq 0}$ be a family of operators on $X$. We say that $\{S(t)\}_{t \geq 0}$ is a $C^{0}$ semigroup on $X$ if $\{S(t)\}_{t \geq 0}$ satisfies the following conditions:

(i) $S(0)=I d$ (the identity),

(ii) $S(t) S(s)=S(t+s)$ for all $t, s \geq 0$,

(iii) $S\left(t_{n}\right) x_{n} \rightarrow S(t) x$ if $t_{n} \rightarrow t$ and $x_{n} \rightarrow x$ in $X$.

Definition 2.2 Let $\{S(t)\}_{t \geq 0}$ be a continuous semigroup on $X$. A subset $\mathcal{A}$ in $X$ is called a global attractor if

(1) $\mathcal{A}$ is invariant, that is, $S(t) \mathcal{A}=\mathcal{A}$ for all $t \geq 0$,

(2) $\mathcal{A}$ is compact in $X$,

(3) $\mathcal{A}$ attracts $S(t) B$ as $t \rightarrow \infty$ for each bounded subset $B$ in $X$.

Now we restate the results (see $[1,11,14]$ etc.) about the well-posedness of the solutions and the existence of the global attractor for (1.1).

Theorem 2.3 Under assumption $(A)$, for any $T>0$ and $\left(u_{0}, u_{1}\right) \in H_{0}^{1}(\Omega) \times L^{2}(\Omega)$, there exists a unique solution of (1.1) such that

$$
\left(u, u_{t}\right) \in C\left([0, T] ; H_{0}^{1}(\Omega) \times L^{2}(\Omega)\right) .
$$

We define the mappings

$$
S(t):\left(u_{0}, u_{1}\right) \rightarrow\left(u(t), u_{t}(t)\right)
$$

for all $t \in R$. By Theorem 2.3 it is easy to see that $\{S(t)\}_{t \geq 0}$ is a $C_{0}$-semigroup in the energy phase space $H_{0}^{1}(\Omega) \times L^{2}(\Omega)$.

Theorem 2.4 Under assumption (A), for any fixed $\beta$, problem (1.1) has a global attractor $\mathcal{A}_{\beta}$ in $H_{0}^{1}(\Omega) \times L^{2}(\Omega)$.

Next, we briefly recall the notions of a Lyapunov function, stable manifolds, unstable manifolds, and the $Z_{2}$ index; see $[3,6,12-14]$ and references therein for more detail. 
Definition 2.5 A Lyapunov function for $S(t)$ on a positively invariant set $X \subset H$ is a continuous function $\Phi: X \rightarrow \mathbb{R}$ such that

(i) for each $u_{0} \in X$, the function $t \mapsto \Phi\left(S(t) u_{0}\right)$ is nonincreasing, and

(ii) if $\Phi(S(\tau) u)=\Phi(u)$ for some $\tau>0$, then $u$ is a fixed point of $S(t)$.

Definition 2.6 A fixed point (or a stationary point, or an equilibrium point) is a point $u_{0} \in H$ such that

$$
S(t) u_{0}=u_{0} \quad \forall t \geq 0 .
$$

If $z$ is a fixed point, and $H$ is the phase space, then we have the following definitions.

Definition 2.7 The stable manifold of $z$ is the set

$$
W^{s}(z)=\left\{u_{0} \in H: S(t) u_{0} \text { is defined for all } t, S(t) u_{0} \rightarrow z \text { as } t \rightarrow \infty .\right\}
$$

Definition 2.8 The unstable manifold of $z$ is the set

$$
W^{u}(z)=\left\{u_{0} \in H: S(t) u_{0} \text { is defined for all } t, S(-t) u_{0} \rightarrow z \text { as } t \rightarrow \infty .\right\}
$$

Denote the class of closed symmetric subsets of $X$ by

$$
\mathbb{A}=\{A \subset X: A \text { is closed, } A=-A\} .
$$

Definition 2.9 Let $A \in \mathbb{A}, A \neq \emptyset$. The Krasnoselskii genus or $Z_{2}$ index $\gamma(A)$ of $A$ is defined by

$$
\gamma(A)=\left\{\begin{array}{l}
\inf \left\{m: \exists h \in C^{0}\left(A, \mathbb{R}^{m} \backslash\{0\}\right), h(-u)=-h(u)\right\} \\
\infty, \quad \text { if }\left\{m: \exists h \in C^{0}\left(A, \mathbb{R}^{m} \backslash\{0\}\right), h(-u)=-h(u)\right\}=\emptyset, \\
\quad \text { in particular, if } 0 \in A,
\end{array}\right.
$$

and $\gamma(\emptyset)=0$.

\section{Main results}

In this section, we consider the property of the global attractor $\mathcal{A}_{\beta}$ of (1.1) and obtain that there are at least $n$ pairs of different fixed points in the global attractor $\mathcal{A}_{\beta}$. We first give two lemmas. Their proofs are similar to those in [9], and we omit them.

Lemma 3.1 For any fixed $\beta$, the semigroup $\{S(t)\}_{t \geq 0}$ associated with the solutions of (1.1) is odd, and the global attractor $\mathcal{A}_{\beta}$ obtained in Theorem 2.4 is symmetric.

Define the energy function

$$
F(\phi)=F\left(u, u_{t}\right)=\int_{\Omega}\left\{\frac{1}{2}\left(|\nabla u|^{2}+\left|u_{t}\right|^{2}\right)-\frac{\lambda}{2}|u|^{2}+\Phi(u)\right\} d x,
$$

where $\phi=\left(u, u_{t}\right)$, and $\Phi(u)=\int_{0}^{u} \varphi(s) d s$ is the primitive function of $\varphi(u)$.

Hereafter, we denote $v=u_{t}$ and $v_{0}=u_{t}(0)=u_{1}$. 
Lemma 3.2 The function $F(\phi)$ defined by (3.1) is an even Lyapunov function on $H_{0}^{1}(\Omega) \times$ $L^{2}(\Omega)$ for the semigroup $\{S(t)\}_{t \geq 0}$.

Theorem 3.3 Suppose assumption $(A)$ holds, let $\mathcal{A}_{\beta}$ be the global attractor of (1.1) for any fixed $\beta$, and let $F$ be the Lyapunov function on $X=H_{0}^{1}(\Omega) \times L^{2}(\Omega)$ for the semigroup $\{S(t)\}_{t \geq 0}$ defined by (3.1). Then for any natural number $n$, there exists $\beta$ large enough, and there exist closed subspaces $X^{+}$and $X^{-}$of $X$ such that:

(i) $X=X^{+}+X^{-}$,

(ii) $\operatorname{dim} X^{-}-\operatorname{codim} X^{+} \geq n$,

(iii) there exist $\alpha>0$ and $\delta>0$ such that $\left.F\right|_{X^{+} \cap \partial B(0, \delta)} \geq \alpha$,

(iv) there exist $R$ and $0<\rho<R$ such that

$$
\left.F\right|_{X-\cap \partial B(0, R)} \inf _{\phi \in \partial B(0, \rho)} F(\phi)<F(0)=0 .
$$

Proof Let $Y=H_{0}^{1}(\Omega)$ and $Z=L^{2}(\Omega)$. Denote by $Y^{+}$the closed subspace of $Y$ spanned by $e_{k}, k=m+j, j=1,2, \ldots$, that is,

$$
Y^{+}=\overline{\operatorname{span}\left\{e_{k}: k=m+j, j=1,2, \ldots\right\}},
$$

where the closure is taken in $Y$. For any natural number $n$, denote

$$
Y^{-}=\overline{\operatorname{span}\left\{e_{k}: k=1,2, \ldots, m+n\right\}} .
$$

Set $X^{-}=Y^{-} \times\{0\}$ and $X^{+}=Y^{+} \times Z$. Then we easily obtain

$$
X=X^{+}+X^{-}
$$

and

$$
\operatorname{dim} X^{-}-\operatorname{codim} X^{+}=n .
$$

In the following, we prove (iii). For any $\phi \in X^{+}$, we have

$$
\|\nabla u\|_{L^{2}(\Omega)}^{2} \geq \lambda_{m+1}\|u\|_{L^{2}(\Omega)}^{2}
$$

By the Sobolev embeddings $H_{0}^{1}(\Omega) \hookrightarrow L^{4}(\Omega)$ and $H_{0}^{1}(\Omega) \hookrightarrow L^{\gamma+2}(\Omega)$ we have

$$
\begin{aligned}
F(\phi)= & \int_{\Omega}\left\{\frac{1}{2}\left(|\nabla u|^{2}+|v|^{2}\right)-\frac{\lambda}{2}|u|^{2}+C u^{4}-\frac{\beta}{\gamma+2}|u|^{\gamma+2}\right\} d x \\
\geq & \frac{1}{2}\|\nabla u\|_{L^{2}(\Omega)}^{2}+\frac{1}{2}\|v\|_{L^{2}(\Omega)}^{2}-\frac{\lambda}{2}\|u\|_{L^{2}(\Omega)}^{2}+C\|u\|_{L^{4}(\Omega)}^{4}-\frac{\beta}{\gamma+2}\|u\|_{L^{\gamma+2}(\Omega)}^{\gamma+2} \\
\geq & \left(\frac{1}{2}-\frac{\lambda}{2 \lambda_{m+1}}\right)\|\nabla u\|_{L^{2}(\Omega)}^{2}+\frac{1}{2}\|v\|_{L^{2}(\Omega)}^{2} \\
& +C\|u\|_{L^{4}(\Omega)}^{4}-\frac{\beta}{\gamma+2} C\|\nabla u\|_{L^{2}}^{\gamma+2}(\Omega),
\end{aligned}
$$


which implies that, for fixed $\beta$ properly large, there exists a constant $\delta>0$ such that, for any $\phi \in X^{+} \cap \partial B(0, \delta)$,

$$
\left.F\right|_{X^{+} \cap \partial B(0, \delta)} \geq \alpha,
$$

where $\alpha$ is a fixed positive constant.

Finally, we prove (iv). For $\phi_{1}=\left(e_{1}, u_{t}\right)$, we have

$$
\begin{aligned}
F\left(\varepsilon \phi_{1}\right) & =\int_{\Omega}\left\{\frac{1}{2} \varepsilon^{2}\left(\left|\nabla e_{1}\right|^{2}+\left|u_{t}\right|^{2}\right)-\frac{\lambda}{2}\left|\varepsilon e_{1}\right|^{2}+\Phi\left(\varepsilon e_{1}\right)\right\} d x \\
& =\int_{\Omega}\left\{\frac{1}{2} \varepsilon^{2}\left(\left|\nabla e_{1}\right|^{2}+\left|u_{t}\right|^{2}\right)-\frac{\lambda}{2}\left|\varepsilon e_{1}\right|^{2}+C \varepsilon^{4}\left|e_{1}\right|^{4}-\frac{\beta}{\gamma+2} \varepsilon^{\gamma+2}\left|e_{1}\right|^{\gamma+2}\right\} d x \\
& =\int_{\Omega}\left\{\frac{1}{2} \varepsilon^{2}\left|u_{t}\right|^{2}+\frac{\lambda_{1}-\lambda}{2}\left|\varepsilon e_{1}\right|^{2}+C \varepsilon^{4}\left|e_{1}\right|^{4}-\frac{\beta}{\gamma+2} \varepsilon^{\gamma+2}\left|e_{1}\right|^{\gamma+2}\right\} d x .
\end{aligned}
$$

For any $\phi \in X_{1}=\left\{u \in X \mid\|\nabla u\|_{L^{2}(\Omega)}+\left\|u_{t}\right\|_{L^{2}(\Omega)}=1\right\}$, by the Sobolev embedding $H_{0}^{1}(\Omega) \hookrightarrow$ $L^{6}(\Omega) \hookrightarrow L^{4}(\Omega) \hookrightarrow L^{\gamma+2}(\Omega) \hookrightarrow L^{2}(\Omega)$ we deduce that there exist positive constants $\sigma_{1}$ and $\sigma_{2}$ such that

$$
\sup _{\phi \in X_{1}}\|u\|_{L^{2}(\Omega)}^{2}=\sigma_{1}, \quad \sup _{\phi \in X_{1}}\|u\|_{L^{\gamma+2}(\Omega)}^{\gamma+2}=(\gamma+2) \sigma_{2} .
$$

Hence we get

$$
\begin{aligned}
F(s \phi) & =\int_{\Omega}\left\{\frac{1}{2} s^{2}+C s^{4}|u|^{4}-\frac{\lambda}{2}|s u|^{2}-\frac{\beta}{\gamma+2} s^{\gamma+2}|u|^{\gamma+2}\right\} d x \\
& \geq-\frac{\lambda}{2} \sigma_{1} s^{2}-\sigma_{2} \beta s^{r} \rightarrow 0 \quad \text { as } s \rightarrow 0 .
\end{aligned}
$$

Combining (3.3) with (3.4), we infer that there exists $\rho \in(0,1)$ such that

$$
-\infty<\inf _{\phi \in \partial B(0, \rho)} F(\phi)<0
$$

for properly large $\beta$. Then, for any $\phi \in X_{2}:=\left\{u \in X^{-}:\|u\|_{H_{0}^{1}(\Omega)}+\left\|u_{t}\right\|_{L^{2}(\Omega)}=\|u\|_{H_{0}^{1}(\Omega)}=1\right\}$, there exists a constant $\theta>0$ such that

$$
\inf _{\phi \in X_{2}}\|u\|_{L^{\gamma+2}(\Omega)}^{\gamma+2}=(\gamma+2) \theta .
$$

Therefore it follows from (3.5) that, for any $\phi \in X_{2}$ and $\mu \in \mathbb{R}$,

$$
\begin{aligned}
F(\mu \phi) & =\int_{\Omega}\left\{\frac{1}{2} \mu^{2}\left(|\nabla u|^{2}+\left|u_{t}\right|^{2}\right)+C \mu^{4}|u|^{4}-\frac{\lambda}{2}|\mu u|^{2}-\frac{\beta}{\gamma+2} \mu^{\gamma+2}|u|^{\gamma+2}\right\} d x \\
& \leq \frac{1}{2} \mu^{2}+C \mu^{4}-\frac{\lambda}{2 \lambda_{1}} \mu^{2}-\beta \mu^{\gamma+2} \theta .
\end{aligned}
$$

We choose $R$ such that $R^{\gamma+2} \theta>\rho^{\gamma+2} \sigma_{2}$. Then for properly large $\beta$, we get

$$
\left.F\right|_{X-\cap \partial B(0, R)}<\inf _{\phi \in \partial B(0, \rho)} F(\phi)<F(0)=0 .
$$


From Theorems 1.1 and 1.2, Lemmas 3.1 and 3.2, and Theorem 3.3, we obtain the following theorem and corollary.

Theorem 3.4 Suppose assumption $(A)$ holds. Let $\mathcal{A}_{\beta}$ be the global attractor of (1.1) for any fixed $\beta$, and let $F$ be a Lyapunov function on $H_{0}^{1}(\Omega) \times L^{2}(\Omega)$ for the semigroup $\{S(t)\}_{t \geq 0}$ defined by (2.1). Then for any natural number $n$, there exists $\beta$ large enough such that

$$
\gamma\left(\mathcal{A}_{\beta} \cap F^{-1}([\alpha, \infty))\right) \geq n
$$

Corollary 3.5 Under the assumptions of Theorem 3.4, for any natural number $n$, there exists $\beta$ large enough such that the semigroup $\{S(t)\}_{t \geq 0}$ possesses at least $n$ pairs of different fixed points in $\mathcal{A}_{\beta} \cap F^{-1}((0, \infty))$.

From [5] we know that any compact set $A$ with fractal $\operatorname{dimension} \operatorname{dim}_{F} A=n$ can be mapped into $\mathbb{R}^{2 n+1}$ by a linear odd Hölder-continuous one-to-one projector. Similar to Corollary 1.1 in [17], we have the following corollary.

Corollary 3.6 Suppose assumption $(A)$ holds. Let $\mathcal{A}_{\beta}$ be the global attractor of (1.1) for any fixed $\beta$. Then

$$
\lim _{\beta \rightarrow \infty} \operatorname{dim}_{F} \mathcal{A}_{\beta}=\infty
$$

\section{Acknowledgements}

The authors wish to express their gratitude to the anonymous referees for their valuable comments and suggestions, which allowed to improve an early version of this work.

\section{Funding}

This work was supported by the NSFC (11701230, 11801227, 11801228), QingLan Project, Jiangsu Overseas visiting scholar Program for University Prominent Young Middle aged Teachers and Presidents and Natural Science Foundation of Jiangsu Province (BK20170308).

\section{Availability of data and materials}

Not applicable.

\section{Competing interests}

The authors declare that they have no competing interests.

\section{Authors' contributions}

All authors contributed equally and significantly in writing this article. All authors read and approved the finial manuscript.

Authors' information

Fengjuan Meng is an associate professor at School of Mathematics and Physics, Jiangsu University of Technology, Changzhou, China. Cuncai Liu and Chang Zhang are assistant professors at School of Mathematics and Physics, Jiangsu University of Technology, Changzhou, China.

\section{Publisher's Note}

Springer Nature remains neutral with regard to jurisdictional claims in published maps and institutional affiliations.

Received: 28 September 2018 Accepted: 7 January 2019 Published online: 11 January 2019

\section{References}

1. Babin, A.V., Vishik, M.l.: Attractors of Evolution Equations. North-Holland, Amsterdam (1992)

2. Ball, J.M.: Global attractors for damped semilinear wave equations. Discrete Contin. Dyn. Syst. 10, 31-52 (2004)

3. Coffman, C.V.: A minimum-maximum principle for a class of non-linear integral equations. J. Anal. Math. 22, 391-419 (1969)

4. Hale, J.K.: Asymptotic Behavior of Dissipative Systems. Am. Math. Soc., Providence (1988)

5. Hunt, B.R., Kaloshin, V.Y.: Regularity of embeddings of infinite-dimensional fractal sets into finite-dimensional spaces. Nonlinearity 12, 1263-1275 (1999) 
6. Krasnoselskii, M.A.: Topological Methods in the Theory of Nonlinear Integral Equations. Macmillan Co., New York (1964)

7. Li, F., You, B., Zhong, C.K.: Multiple equilibrium points in global attractors for some $p$-Laplacian equations. Appl. Anal. 97(9), 1591-1599 (2018)

8. Meng, F.J, Wang, Y.H., Liu, C.C.: Multiple equilibrium points in global attractor for the strongly damped wave equations. J. Donghua Univ. (Eng. Ed.) 34(3), 448-452 (2017)

9. Meng, F.J., Zhong, C.K.: Multiple equilibrium points in global attractor for the weakly damped wave equation with critical exponent. Discrete Contin. Dyn. Syst., Ser. B 19(1), 217-230 (2014)

10. Miranville, A., Zelik, S.: Attractors for dissipative partial differential equations in bounded and unbounded domains. In: Handbook of Differential Equations: Evolutionary Equations, vol. IV, pp. 103-200. Elsevier, Amsterdam (2008)

11. Pata, V., Zelik, S.: A remark on the damped wave equation. Commun. Pure Appl. Anal. 5(3), 611-616 (2006)

12. Robinson, J.C.: Infinite-Dimensional Dynamical Systems, an Introduction to Dissipative Parabolic PDEs and the Theory of Global Attractors. Cambridge University Press, Cambridge (2001)

13. Struwe, M.: Variational Methods. Springer, Berlin (2000)

14. Temam, R.: Infinite-Dimensional Systems in Mechanics and Physics. Springer, New York (1997)

15. You, B., Li, F., Zhong, C.K.: The existence of multiple equilibrium points in a global attractor for some $p$-Laplacian equation. J. Math. Anal. Appl. 418(2), 626-637 (2014)

16. Zhang, J., Zhong, C.K., You, B.: The existence of multiple equilibrium points in global attractors for some symmetric dynamical systems II. Nonlinear Anal., Real World Appl. 36, 44-55 (2017)

17. Zhong, C.K., Niu, W.S.: On the $Z_{2}$ index of the global attractor for a class of $p$-Laplacian equations. Nonlinear Anal. 73 3698-3704 (2010)

18. Zhong, C.K., You, B., Yang, R.: The existence of multiple equilibrium points in global attractors for some symmetric dynamical systems. Nonlinear Anal., Real World Appl. 19, 31-44 (2014)

\section{Submit your manuscript to a SpringerOpen ${ }^{\circ}$ journal and benefit from:}

- Convenient online submission

- Rigorous peer review

- Open access: articles freely available online

- High visibility within the field

- Retaining the copyright to your article

Submit your next manuscript at $\boldsymbol{\nabla}$ springeropen.com 